\title{
Risk calculations for cystic fibrosis in neonatal screening by immunoreactive trypsinogen and CFTR mutation tests
}

Shuji Ogino, $M D, P h D^{1-3}$, Pamela Flodman, $M S c, M S^{4}$, Robert B. Wilson, $M D, P h D^{5}$, Bert Gold, $P h D^{6}$, and Wayne W. Grody, $\mathrm{MD}, \mathrm{PhD}^{7}$

\begin{abstract}
Purpose: Although neonatal screening (or newborn screening) for cystic fibrosis (CF) is commonly practiced, systematic methods for accurate risk calculations are currently lacking. Methods and Results: We evaluated characteristics of the immunoreactive trypsinogen (IRT) test using the published data. The probability that a neonate has a positive IRT test, if the neonate is affected, a carrier, or a noncarrier, is $\approx 1,0.041$, or 0.011 , respectively. We provide methods to calculate genetic risks for a variety of commonly encountered scenarios in which neonates are positive by the IRT test. Conclusion: Our Bayesian methods permit CF disease probabilities to be calculated accurately, taking into account all relevant information. Genet Med 2005:7(5):317-327.
\end{abstract}

Key Words: Bayesian, CFTR, cystic fibrosis, genetic counseling, screening

Cystic fibrosis (CF; OMIM no. 219700) is the most common severe autosomal recessive disorder in Caucasians, affecting $\approx$ 1 in 2500 live births, and with a carrier frequency of $\approx 1$ in $25 .{ }^{1}$ CF is caused by mutations in the cystic fibrosis transmembrane conductance regulator (CFTR) gene [OMIM no. 602421; 219700 (CF); CFTR mutation database, http://www.genet.sickkids.on.ca/cftr/]. More than 1000 different CFTR variants have been reported, with allele frequencies varying by ethnic group. Risk assessment is an essential component of genetic counseling and testing, and Bayesian analysis plays a central role in complex risk calculations. ${ }^{2-5}$ We previously developed Bayesian methods to calculate the risk of an autosomal recessive disease when only one or no mutation is detected in the disease gene, and another, independent risk factor is present (e.g., fetal echogenic bowel as a risk factor for CF). ${ }^{6}$ Our methods are useful in the setting of CF carrier screening and prenatal testing for CFTR mutations. ${ }^{6}$ We also developed methods to calculate $\mathrm{CF}$ disease and carrier risks in a variety of prenatal

\footnotetext{
From the ${ }^{1}$ Department of Pathology, Brigham and Women's Hospital, Boston, Massachusetts; ${ }^{2}$ Department of Medical Oncology, Dana-Farber Cancer Institute, Boston, Massachusetts; ${ }^{3}$ Harvard Medical School, Boston, Massachusetts; ${ }^{4}$ Department of Pediatrics, University of California Irvine, Irvine, California, ${ }^{5}$ Department of Pathology and Laboratory Medicine, University of Pennsylvania Medical Center, Philadelphia, Pennsylvania; ${ }^{6}$ Human Genetics Section, Laboratory of Genomic Diversity, National Cancer Institute at Frederick, Frederick, Maryland; and the ${ }^{7}$ Departments of Pathology and Laboratory Medicine, Human Genetics, and Pediatrics, UCLA School of Medicine, Los Angeles, California.

The content of this publication does not necessarily reflect the views or policies of the Department of Health and Human Services, nor does mention of trade names, commercial products, or organizations imply endorsement by the U.S. Government.

Shuji Ogino, MD, PhD, Department of Pathology, Brigham and Women's Hospital, Harvard Medical School, 75 Francis St., Boston, MA 02115, shuji_ogino@dfci.harvard.edu.

Received: December 6, 2004.

Accepted: January 7, 2005.
}

DOI: 10.1097/01.GIM.0000162871.68167.8A and carrier screening scenarios that create common, difficult problems. $^{7}$

Neonatal screening for cystic fibrosis is commonly performed as a part of expanded neonatal screening, and usually consists of the immunoreactive trypsinogen (IRT) test followed by CFTR mutation testing if the IRT test is positive. ${ }^{8}$ However, systematic methods are lacking for accurately calculating CF risks after a positive IRT test result and various other CFTR test results are obtained. This is because there are considerable differences in CFTR allele frequencies among different ethnic groups, ${ }^{9,10}$ and published data regarding IRT assay characteristics are complex. ${ }^{11-14}$ In this article, we evaluated published data to calculate the assay characteristics of the IRT test, and provide Bayesian methods for accurate CF risk calculations using concrete scenarios. Our Bayesian methods allow CF disease probabilities to be calculated accurately, taking into account all relevant information. These methods can be modified for many different scenarios, including scenarios concerning other autosomal recessive disorders. We also discuss the utility of sweat chloride testing after neonatal screening.

\section{METHODS AND RESULTS}

There are three key steps to performing a Bayesian risk analysis. First, the problem must be set out in a format that allows the necessary calculations to be specified. One approach is to use probability tables, as presented and illustrated in our previous articles ${ }^{6,7}$; an alternative is to graphically depict all pedigree structures and other available information, as in the unified approach described by Hodge. ${ }^{15}$ Second, the appropriate prior probabilities and conditional probabilities must be determined and included in the table or pedigree. Probabilities relevant for Bayesian analysis in the setting of newborn screen- 
ing for CF are derived and provided below and in Table 1. The third step involves the mathematical calculation of joint and posterior probabilities.

\section{Frequencies of positive IRT tests among CF affected, carrier, and noncarrier neonates}

The frequencies of positive IRT tests among CF affected, carrier, and noncarrier newborns were calculated using a single large study. ${ }^{14}$ All other published studies on the frequencies of positive IRT tests among newborns did not determine the frequencies of positive IRT tests separately among CF affected, carrier, and noncarrier newborns. ${ }^{11-13,16}$ Figure 1 illustrates the numbers derived from the data of Scotet et al. ${ }^{14}$ to calculate each conditional probability that a newborn has hypertrypsinogenemia if the newborn is affected by $\mathrm{CF}$, a carrier, or a noncarrier. In the study by Scotet et al., ${ }^{14}$ a cut-off level of IRT was set as $600 \mu \mathrm{g} / \mathrm{L}$, and $60 \mathrm{CF}$ affected newborns were detected among 160,019 newborns screened, leading to a disease allele frequency of $0.0194\left(q^{2}=60 / 160,019\right)$ assuming Hardy-Weinberg equilibrium. Using the $2 p q(=0.0380)$ carrier frequency, approximately 6,077 newborns were carriers among 160,019 newborns. Among 1,964 hypertrypsinogenemic newborns, 213 were found to be carriers with mutations detected by analysis of three exons (exons 7, 10, and 11, which contain $85 \%$ of CFTR mutations in the Brittany region). ${ }^{14}$ Thus, approximately $250(=213 \times 1 / 0.85)$ carriers and approximately 1,654 $(=1,964-60-250)$ noncarriers were present among the 1964 hypertrypsinogenemic newborns. The number of CF newborns among newborns with IRT levels within normal limits was not determined. ${ }^{14}$ However, the number of CF newborns with normal IRT levels should be small, considering that the CF incidence of $60 / 160,019(=1 / 2,667)$ in this study was so close to the $1 / 2,500$ incidence widely used as the incidence of CF among non-Hispanic Caucasians. ${ }^{1}$ Therefore, the probability that a CF affected, carrier, or noncarrier newborn has hypertrypsinogenemia is estimated to be $1(=60 / 60), 0.041$ ( $=$ $250 / 6,077)$, or $0.011[=1654 /(160,019-60-6,077)]$, respectively (Fig. 1). We can use these probabilities as conditional probabilities in our Bayesian analyses to calculate CF risks for newborns with a positive IRT test.

Table 1

Summary of CF carrier frequencies, overall mutation detection rates by the ACMG panel, and frequencies of major mutations for each major ethnic group (adapted from Watson et al. ${ }^{10}$ and Richards et al. ${ }^{1}$ )

\begin{tabular}{|c|c|c|c|c|}
\hline Ethnic group & $\begin{array}{l}\text { CF carrier } \\
\text { frequency }\end{array}$ & $\begin{array}{l}\text { Overall mutation detection } \\
\text { rate by the ACMG CFTR } \\
23 \text {-mutation panel }{ }^{10}\end{array}$ & $\begin{array}{l}\text { Fraction of F508del } \\
\text { among all disease } \\
\text { alleles }\end{array}$ & Other major mutations (fraction) ${ }^{a}$ \\
\hline \multirow{5}{*}{$\begin{array}{c}\text { Non-Hispanic } \\
\text { Caucasian }\end{array}$} & $1 / 25$ & $88.29 \%$ & $72.42 \%$ & G542X $(2.28 \%)$ \\
\hline & & & & G551D (2.25\%) \\
\hline & & & & $621+1 \mathrm{G}>\mathrm{T}(1.57 \%)$ \\
\hline & & & & W1282X (1.50\%) \\
\hline & & & & N1303K (1.27\%) \\
\hline \multirow[t]{4}{*}{ Ashkenazi Jewish } & $1 / 25$ & $94.04 \%$ & $31.41 \%$ & W1282X (45.92\%) \\
\hline & & & & G542X $(7.55 \%)$ \\
\hline & & & & $3849+10 \mathrm{kbC}>\mathrm{T}(4.77 \%)$ \\
\hline & & & & N1303K (2.78\%) \\
\hline \multirow[t]{6}{*}{ African American } & $1 / 65$ & $64.46 \%$ & $44.07 \%$ & $3120+1 \mathrm{G}>\mathrm{A}(9.57 \%)$ \\
\hline & & & & $\mathrm{R} 553 \mathrm{X}(2.32 \%)$ \\
\hline & & & & I507del (1.87\%) \\
\hline & & & & G542X (1.45\%) \\
\hline & & & & G551D (1.21\%) \\
\hline & & & & $621+1 \mathrm{G}>\mathrm{T}(1.11 \%)$ \\
\hline \multirow[t]{5}{*}{ Hispanic Caucasian } & $1 / 46$ & $71.72 \%$ & $54.38 \%$ & G542X (5.10\%) \\
\hline & & & & R553X (2.81\%) \\
\hline & & & & R334W (1.78\%) \\
\hline & & & & N1303K (1.66\%) \\
\hline & & & & $3849+10 \mathrm{kbC}>\mathrm{T}(1.57 \%)$ \\
\hline \multirow[t]{2}{*}{ Asian American } & $1 / 90$ & $48.93 \%$ & $38.95 \%$ & $3849+10 \mathrm{kbC}>\mathrm{T}(5.31 \%)$ \\
\hline & & & & G551D (3.15\%) \\
\hline
\end{tabular}



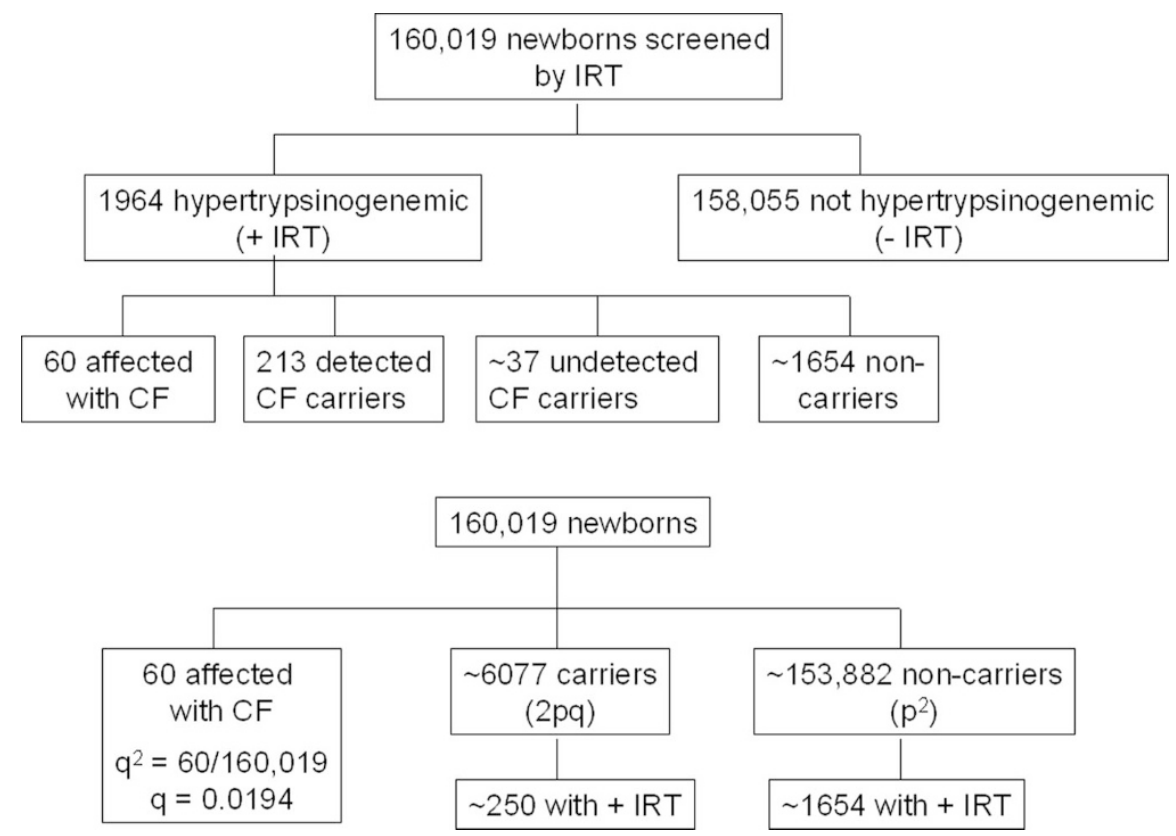

Fig. 1. Analysis of characteristics of IRT test.

\section{Bayesian analysis to calculate CF risks for neonates with a positive IRT test}

A fraction of each major CFTR disease allele among all CFTR disease alleles and a mutation detection rate are summarized for each of five major ethnic groups (Table 1). We assumed that CFTR mutation testing detects the 23 CFTR mutations designated for cancer screening by the American College of Medical Genetics (ACMG), ${ }^{10}$ which is a slight modification of the original 25 CFTR mutation panel. ${ }^{1,17}$ We used carrier frequencies for non-Hispanic Caucasian (1/25), Ashkenazi Jewish (1/25), African American (1/65), Hispanic Caucasian (1/46), and Asian American (1/90).1,6 We used mutation detection rates and the fractions of major mutations for major ethnic groups as reviewed previously. ${ }^{10}$ In particular, we used mutation detection rates of $0.88,0.94,0.64$, and 0.72 for nonHispanic Caucasian, Ashkenazi Jewish, African American, and Hispanic Caucasian populations, respectively (Table 1). One can modify our methods with regard to mutation detection rates, as long as the fraction of each mutation in a CFTR testing panel in question among all CFTR disease alleles is known. The ethnicity-specific fractions of the common F508del mutation [C.1522_1524 del TTT (p.Phe508del)] among all CFTR mutations are $0.72,0.31,0.44$, and 0.54 for non-Hispanic Caucasian, Ashkenazi Jewish, African American, and Hispanic Caucasian populations, respectively (Table 1 ).

\section{Scenario 1. Non-Hispanic Caucasian neonate with a positive IRT test, CFTR mutation testing on the neonate pending or not performed, and no family history or CFTR mutation testing on parents or other relatives}

There is usually a time delay between the time of a positive IRT test result and the time of the completion of CFTR mutation testing. What is the risk that the neonate is affected before
CFTR mutation testing, or if CFTR mutation testing is not performed? Suppose a non-Hispanic Caucasian neonate has a positive result in the IRT test; the Bayesian analysis for the CF risk of the neonate is shown in Table 2. Before the IRT test is performed, the probability that the neonate is affected, a CF carrier, or a noncarrier is $0.0004,0.04$, or 0.96 , respectively. The conditional probability that the neonate is positive by the IRT test, if the neonate is affected, a carrier, or a noncarrier, is $1,0.041$, or 0.011 , respectively. The joint probability that the neonate is affected, a carrier, or a noncarrier, and that the neonate shows a positive IRT result, is the product of each prior probability and the conditional probability for each column (i.e., "Affected," "Carrier," or "Noncarrier"). To obtain the posterior probability that the neonate is affected given a positive IRT test, one can divide the joint probability that the neonate is affected and shows a positive IRT test, by the probability that the neonate shows a positive IRT test regardless of genotype (i.e., the sum of all of the joint probabilities), yielding 0.032 . The posterior probability that the neonate is a carrier or a noncarrier given a positive IRT test is the joint probability for

Table 2

Bayesian analysis for a non-Hispanic Caucasian neonate with a positive IRT test (Scenario 1) ${ }^{a}$

\begin{tabular}{llll}
\hline Hypothesis for the neonate & Affected & Carrier & Noncarrier \\
\hline $\begin{array}{l}\text { Prior probability } \\
\begin{array}{l}\text { Conditional probability of a } \\
\text { positive IRT test result }\end{array}\end{array}$ & 0.0004 & 0.04 & 0.96 \\
$\begin{array}{l}\text { Joint probability } \\
\text { Posterior probability }\end{array}$ & 0.0004 & 0.041 & 0.011 \\
\hline
\end{tabular}

${ }^{a}$ CFTR mutation testing has not been performed on the neonate, parents, or other relatives. 
each column divided by the sum of all of the joint probabilities ( 0.13 or 0.84 , respectively). After CFTR mutation testing is performed, Table 2 will be modified to incorporate the results of CFTR mutation testing (see Scenarios 2 and 3).

\section{Scenario 2. Non-Hispanic Caucasian neonate with a positive IRT test, one identifiable CFTR mutation (F508del), and no family history of CF}

As in Scenario 1, the probability (before IRT and CFTR mutation testing) that the neonate is affected, a CF carrier, or a noncarrier is $0.0004,0.04$, or 0.96, respectively. The Bayesian analysis for this scenario is shown in Table 3 . The challenge in this case is in the second step of the process, which is determining the conditional probability of finding a single identifiable mutation, for each column. We demonstrate the process for the first column: if the neonate is affected, then both parents are known to be carriers, and there are two possibilities, which are that the mutation from parent $\mathrm{A}(\mathrm{Da})$ is F508del with the mutation from parent $\mathrm{B}(\mathrm{Db})$ undetectable, or the mutation from parent $\mathrm{B}(\mathrm{Db})$ is F508del with the mutation from parent A (Da) undetectable. The probability in this ethnic group that a CFTR mutation is F508del or undetectable can be determined from Table 1 . Thus, the conditional probability that the neonate has only one identifiable mutation F508del if affected is the fraction of the identified mutation (among all CFTR mutations) in the father's ethnic background population multiplied by $(1-$ mutation detection rate for the mother's ethnic background population), plus the fraction of the identified mutation (among all CFTR mutations) in the mother's ethnic background population multiplied by ( 1 - mutation detection rate for the father's ethnic background population) (i.e., $0.72 \times 0.12+0.12 \times 0.72=0.1728$ ). For the second column in Table 3 , the conditional probability that the neonate has one identifiable mutation if the neonate is a carrier is the fraction of the identified mutation in the parents' background population (i.e., 0.72). Clearly, for the third column in Table 3, the conditional probability that the neonate has one identifiable mutation F508del if the neonate is a noncarrier is 0 . By applying the third step of carrying out the necessary mathematical calcula-

Table 3

Bayesian analysis for a non-Hispanic Caucasian neonate with a positive IRT test and one identifiable CFTR mutation (Scenario 2) ${ }^{a}$

\begin{tabular}{lccc}
\hline Hypothesis for the neonate & Affected & Carrier & Noncarrier \\
\hline $\begin{array}{l}\text { Prior probability } \\
\begin{array}{l}\text { Conditional probability of one } \\
\text { identifiable mutation }\end{array}\end{array}$ & $\begin{array}{c}0.72 \times 0.12 \\
+0.12 \times 0.72\end{array}$ & 0.04 & 0.96 \\
$\begin{array}{l}\text { Conditional probability of a } \\
\text { positive IRT test result }\end{array}$ & 1 & 0.041 & 0 \\
$\begin{array}{l}\text { Joint probability } \\
\text { Posterior probability }\end{array}$ & 0.000069 & 0.0012 & 0 \\
\hline
\end{tabular}

${ }^{a}$ CFTR mutation testing has not been performed on the parents or other relatives. tions, it is determined that the posterior probability that the neonate is affected is 0.056 .

\section{Scenario 3. Non-Hispanic Caucasian neonate with a positive IRT test, no identifiable CFTR mutation, and no family history of CF}

This scenario is probably one of the most common counseling scenarios in CF newborn screening. As in Scenarios 1 and 2, the prior probability that the neonate is affected, a CF carrier, or a noncarrier is $0.0004,0.04$, or 0.96 , respectively. The Bayesian analysis for this scenario is shown in Table 4 . The conditional probability that the neonate has no identifiable mutation, if the neonate is affected, a CF carrier, or a noncarrier, is (1 - mutation detection rate for the father's ethnic background population) multiplied by ( 1 - mutation detection rate for the mother's ethnic background population), (1 mutation detection rate for the parent's ethnic background population), or 1 , respectively. The posterior probability that the neonate is affected or a carrier is 0.00054 or 0.018 , respectively.

\section{Scenario 4. Neonate with a non-Hispanic Caucasian parent A, an Ashkenazi Jewish parent B, a positive IRT test, one identifiable CFTR mutation (F508del), and no family history of CF}

For this scenario, we cannot begin with the prior probabilities of particular hypotheses for the fetus as in Scenarios 2 and 3. Instead, we must begin with the prior probabilities of particular hypotheses for the parents before the IRT test and the CFTR mutation testing are performed. This is because the ethnicities of parent A and parent B are different, as are the CFTR allelic spectra in these ethnicities, so we need to follow paternally-derived and maternally-derived mutations separately if any mutation exists in either parent. The Bayesian analysis for this scenario is shown in Table 5, which is based on Scenario 1 in Ogino et al. ${ }^{6}$ For simplicity, columns for which the neonate is a noncarrier have not been included in this table; this is because the joint probability for each of these columns will be 0 , because the conditional probability that a noncarrier neonate has a single identifiable mutation is 0 . Before the IRT test and CFTR mutation testing are performed, the probability that parent $A$ or parent $B$ is a CF carrier is 0.04 or 0.04 , respectively.
Table 4

Bayesian analysis for a non-Hispanic Caucasian neonate with a positive IRT test and no detectable CFTR mutation (Scenario 3) ${ }^{a}$

\begin{tabular}{llll}
\hline Hypothesis & \multicolumn{1}{c}{ Affected } & Carrier & Noncarrier \\
\hline $\begin{array}{l}\text { Prior probability } \\
\begin{array}{l}\text { Conditional probability of no } \\
\text { detectable mutation }\end{array}\end{array}$ & 0.0004 & 0.04 & 0.96 \\
$\begin{array}{l}\text { Conditional probability of } \\
\text { positive IRT test }\end{array}$ & 1 & $0.12 \times 1$ & 1 \\
$\begin{array}{l}\text { Joint probability } \\
\text { Posterior probability }\end{array}$ & 0.0000058 & 0.041 & 0.011 \\
\hline
\end{tabular}

${ }^{a}$ CFTR mutation testing has not been performed on the parents or other relatives. 
For each column, the conditional probability given the parental genotypes that the neonate is affected, a carrier with a mutation from parent $\mathrm{A}$, or a carrier with a mutation from parent $\mathrm{B}$ can be calculated by applying simple Mendelian ratios. For example, if both parents are carriers, these three conditional probabilities are $1 / 4,1 / 4$, or $1 / 4$, respectively. The conditional probabilities of a positive IRT test result given each column are incorporated in the next row of the table, as before. Next, we incorporate the information that the neonate has one identifiable mutation F508del. If the neonate is affected, there are two possibilities: either the mutation from parent $\mathrm{A}(\mathrm{Da})$ is F508del and the mutation from parent $\mathrm{B}(\mathrm{Db})$ is undetectable, or the mutation from parent $B(D b)$ is F508del and the mutation from parent $\mathrm{A}(\mathrm{Da})$ is undetectable. Columns $\mathrm{A}$ and $\mathrm{B}$ in Table 5 correspond to these two possibilities. The posterior probability that the neonate is affected is the sum of the posterior probabilities of columns A and B, or 0.037 .

Scenario 4 provides an example of how the unified approach described by Hodge ${ }^{15}$ can be used to perform Bayesian risk calculations (Fig. 2). In this approach, we identify all possible pedigree configurations that could lead to a neonate with a positive IRT test and one F508del ( $\Delta$ F508) mutation. The six possible configurations are depicted in a schematic way rather than in a table. The relevant probabilities are drawn directly onto the pedigree. The joint probability of each configuration is determined, allowing for the calculation of posterior risk that the neonate is affected. The main advantage of this method is that it allows the identification of all possible pedigree permutations with relative ease, and that it is clear that the Bayesian analysis begins at the top of the pedigree.

\section{Scenario 5. Neonate of a Non-Hispanic Caucasian parent $A$ and an African American parent B, with a positive IRT test, no identifiable CFTR mutation, and no family history of CF}

Before the IRT test and CFTR mutation testing are performed, the probability that parent $A$ or parent $B$ is a CF carrier is $1 / 25$ or $1 / 65$, respectively. The Bayesian analysis for this scenario is shown in Table 6, which is based on Scenario 5 in Ogino et al. ${ }^{6}$ The conditional probabilities that the neonate has no identifiable mutation given that the neonate is affected, a carrier of a mutation inherited from parent $\mathrm{A}$, a carrier of a mutation inherited from parent $\mathrm{B}$, or a noncarrier are calculated from Table 1 (as in Scenario 3). The conditional probability that the neonate has no identifiable mutation if affected is (1 - mutation detection rate for parent A's ethnic background population) multiplied by ( $1-$ mutation detection rate for

Table 5

Bayesian analysis for a neonate born to non-Hispanic Caucasian and Ashkenazi Jewish parents (Scenario 4$)^{a}$

\begin{tabular}{|c|c|c|c|c|c|c|c|}
\hline \multirow{3}{*}{$\begin{array}{l}\begin{array}{l}\text { Non-Hispanic } \\
\text { Caucasian parent A }\end{array} \\
\text { Prior probability } \\
\text { Ashkenazi Jewish } \\
\quad \text { parent B }\end{array}$} & \multicolumn{4}{|c|}{ Carrier (Da) } & & \multicolumn{2}{|c|}{ Noncarrier } \\
\hline & \multicolumn{4}{|c|}{0.04} & & \multicolumn{2}{|c|}{0.96} \\
\hline & \multicolumn{4}{|c|}{ Carrier (Db) } & Noncarrier & Carrier (Db) & Noncarrier \\
\hline Prior probability & \multicolumn{4}{|c|}{0.04} & 0.96 & 0.04 & 0.96 \\
\hline \multicolumn{8}{|l|}{$\begin{array}{l}\text { Conditional } \\
\text { probabilities }\end{array}$} \\
\hline \multirow{2}{*}{$\begin{array}{l}\text { Neonate with one } \\
\text { mutation } \\
\text { detected }\end{array}$} & \multicolumn{2}{|c|}{ Affected $(\mathrm{Da}+\mathrm{Db})$} & Carrier (Da) & Carrier (Db) & Carrier (Da) & Carrier (Db) & $\cdots$ \\
\hline & \multicolumn{2}{|c|}{0.25} & 0.25 & 0.25 & 0.5 & 0.5 & 0 \\
\hline Positive IRT test & \multicolumn{2}{|c|}{1} & 0.041 & 0.041 & 0.041 & 0.041 & 0.011 \\
\hline \multirow{2}{*}{$\begin{array}{l}\text { One mutation } \\
\text { identified }\end{array}$} & $\mathrm{Da}$ & $\mathrm{Db}$ & $\mathrm{Da}$ & $\mathrm{Db}$ & $\mathrm{Da}$ & $\mathrm{Db}$ & $\cdots$ \\
\hline & 0.72 & 0.31 & 0.72 & 0.31 & 0.72 & 0.31 & $\cdots$ \\
\hline \multirow{2}{*}{$\begin{array}{l}\text { No other } \\
\text { mutation } \\
\text { detected }\end{array}$} & $\mathrm{Db}$ & $\mathrm{Da}$ & $\mathrm{N}$ & $\mathrm{N}$ & $\mathrm{N}$ & $\mathrm{N}$ & $\mathrm{N}$ \\
\hline & 0.060 & 0.12 & 1 & 1 & 1 & 1 & 1 \\
\hline Joint probability & $1.7 \times 10^{-5}$ & $1.5 \times 10^{-5}$ & $1.2 \times 10^{-5}$ & $5.1 \times 10^{-6}$ & $5.7 \times 10^{-4}$ & $2.4 \times 10^{-4}$ & 0 \\
\hline Posterior probability & 0.020 & 0.017 & 0.014 & 0.0059 & 0.66 & 0.28 & 0 \\
\hline Column & A & B & $\mathrm{C}$ & $\mathrm{D}$ & E & F & G \\
\hline
\end{tabular}

${ }^{a}$ Neonate has a positive IRT test result, and has been found to have one detectable mutation (heterozygous). Neither parent has been tested, nor does either have a family history of CF.

$\mathrm{Da}$, disease allele derived from parent $\mathrm{A} ; \mathrm{Db}$, disease allele derived from parent $\mathrm{B} ; \mathrm{N}$, normal allele. 


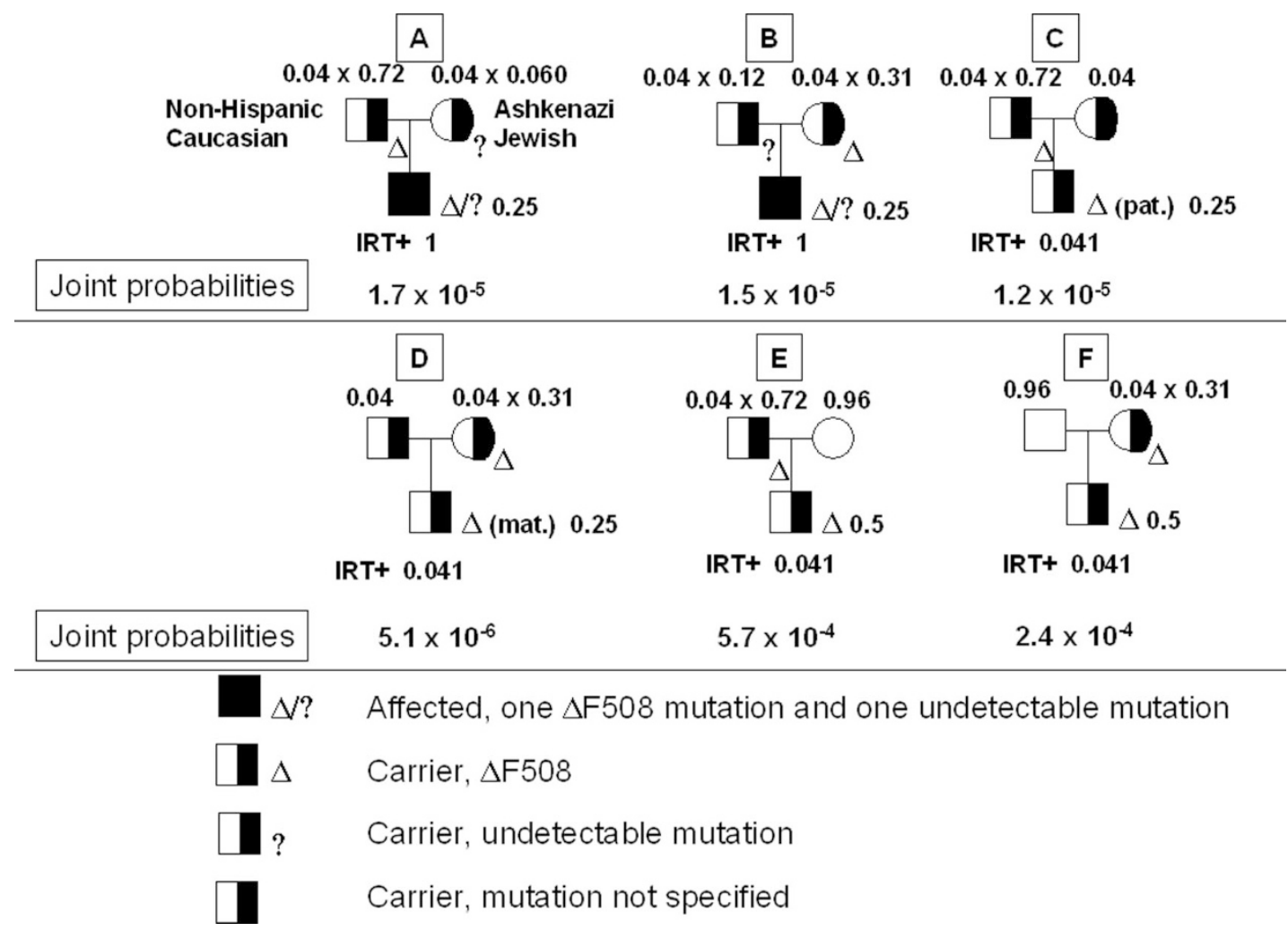

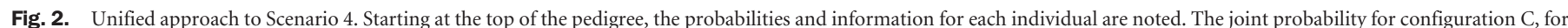

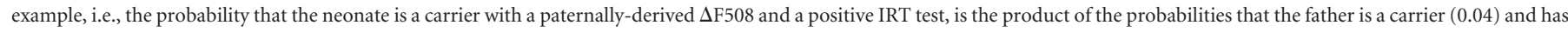

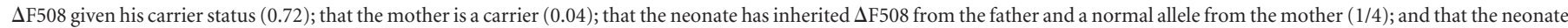
tests positive for IRT given his or her carrier status (0.041).

Table 6

Bayesian analysis for a neonate born to non-Hispanic Caucasian and African American parents (Scenario 5) ${ }^{a}$

\begin{tabular}{|c|c|c|c|c|c|c|c|c|c|}
\hline Non-Hispanic Parent A & \multicolumn{6}{|c|}{ Carrier (Da) } & \multicolumn{3}{|c|}{ Noncarrier } \\
\hline Prior probability & \multicolumn{6}{|c|}{$1 / 25$} & \multicolumn{3}{|c|}{$24 / 25$} \\
\hline African American Parent B & \multicolumn{4}{|c|}{ Carrier (Db) } & \multicolumn{2}{|c|}{ Noncarrier } & \multicolumn{2}{|c|}{ Carrier (Db) } & Noncarrier \\
\hline Prior probability & \multicolumn{4}{|c|}{$1 / 65$} & \multicolumn{2}{|c|}{$64 / 65$} & \multicolumn{2}{|c|}{$1 / 65$} & $64 / 65$ \\
\hline \multicolumn{10}{|l|}{ Conditional probabilities } \\
\hline Neonate (mutation) & $\begin{array}{c}\text { Affected } \\
(\mathrm{Da}+\mathrm{Db})\end{array}$ & $\begin{array}{c}\text { Carrier } \\
(\mathrm{Da})\end{array}$ & $\begin{array}{l}\text { Carrier } \\
(\mathrm{Db})\end{array}$ & Noncarrier & $\begin{array}{c}\text { Carrier } \\
\text { (Da) }\end{array}$ & Noncarrier & $\begin{array}{c}\text { Carrier } \\
\text { (Db) }\end{array}$ & Noncarrier & Noncarrier \\
\hline & $1 / 4$ & $1 / 4$ & $1 / 4$ & $1 / 4$ & $1 / 2$ & $1 / 2$ & $1 / 2$ & $1 / 2$ & 1 \\
\hline Positive IRT test & 1 & 0.041 & 0.041 & 0.011 & 0.041 & 0.011 & 0.041 & 0.011 & 0.011 \\
\hline No mutation detected & $0.12 \times 0.36$ & 0.12 & 0.36 & 1 & 0.12 & 1 & 0.36 & 1 & 1 \\
\hline Joint probability & $6.6 \times 10^{-6}$ & $7.6 \times 10^{-7}$ & $2.3 \times 10^{-6}$ & $1.7 \times 10^{-6}$ & $9.7 \times 10^{-5}$ & $2.2 \times 10^{-4}$ & $1.1 \times 10^{-4}$ & $8.1 \times 10^{-5}$ & $1.0 \times 10^{-2}$ \\
\hline Posterior probability & $\begin{array}{c}0.00061 \\
(=1 / 1600)\end{array}$ & 0.000069 & 0.00021 & 0.00016 & 0.0089 & 0.020 & 0.010 & 0.0074 & 0.95 \\
\hline Column & A & B & $\mathrm{C}$ & $\mathrm{D}$ & $\mathrm{E}$ & $\mathrm{F}$ & G & $\mathrm{H}$ & I \\
\hline
\end{tabular}

${ }^{a}$ Neonate has a positive IRT test result, and has been found to have no detectable mutation.

$\mathrm{Da}$, disease allele derived from parent $\mathrm{A} ; \mathrm{Db}$, disease allele derived from parent $\mathrm{B} ; \mathrm{N}$, normal allele.

parent B's ethnic background population). The conditional probability that the neonate has no identifiable mutation if the neonate is a carrier with a mutation derived from parent $\mathrm{A}$ or $\mathrm{B}$ is $(1$ - mutation detection rate for parent A's ethnic background population) or $(1-$ mutation detection rate for parent B's ethnic background population), respectively. The posterior 
probability that the neonate is affected is $0.00061(=1 / 1600)$. The posterior carrier probability of the neonate is the sum of the posterior probabilities of columns B, C, E, and G, or 0.019 .

\section{Scenario 6. Neonate of a Non-Hispanic Caucasian parent A, a Hispanic American parent B, with a positive IRT test and one identifiable CFTR mutation (F508del); parent A with a family history of CF, parent B with no family history of CF, and neither parent tested}

Because parent A has a family history of CF, prenatal testing and/or carrier screening should have been offered. Unfortunately, however, this scenario is not uncommon. Suppose that parent $\mathrm{A}$ has an affected nephew who has not been tested, and that neither of the parents has been tested. Before the IRT test and CFTR mutation testing are performed, the probability that parent A or B is a CF carrier is $1 / 2$ or $1 / 46$, respectively. The Bayesian analysis and the unified approach (Hodge ${ }^{15}$ ) are shown in Table 7 and Figure 3. This example is based on Scenario 1 in Ogino et al. ${ }^{6}$ There is a small possibility that each parent of parent $\mathrm{A}$ has a disease allele, and that parent $\mathrm{A}$ inherited the disease allele different from the one present in the affected nephew. Including this possibility, parent A's carrier probability is 0.5025 , representing only a $0.5 \%$ increase from the 0.5 carrier probability that derives from the mutations present in the affected nephew (calculations not shown). Our approach starting from parent $\mathrm{A}$ is straightforward and the results are essentially the same as those by an approach starting from parents of parent A (calculations not shown). The prior probability that parent $\mathrm{A}$ is a carrier or a noncarrier is $1 / 2$ or $1 / 2$, respectively. The prior probability that parent $B$ is a carrier or a noncarrier is $1 / 46$ or $45 / 46$, respectively. The conditional probability that the neonate is affected, a carrier with a mutation from parent $\mathrm{A}$, or a carrier with a mutation from parent $\mathrm{B}$, if both parents are carriers, is $1 / 4,1 / 4$, or $1 / 4$, respectively. The conditional probability that the neonate is a carrier with a mutation from parent $\mathrm{A}$ (or parent $\mathrm{B}$ ), if parent $\mathrm{A}$ is a carrier (or a noncarrier) and parent $B$ is a noncarrier (or a carrier), is $1 / 2$ (or $1 / 2$, respectively). If the neonate is affected, there are two possibilities: either the mutation from parent A (Da) is F508del and the mutation from parent $\mathrm{B}(\mathrm{Db})$ is undetectable, or the mutation from parent $\mathrm{B}(\mathrm{Db})$ is F508del and mutation from parent $\mathrm{A}(\mathrm{Da})$ is undetectable. Thus, the conditional probability that the neonate has one identifiable mutation if affected is the sum of the fraction of that identified mutation in parent A's ethnic background population multiplied by $(1-$ mutation detection rate for parent B's ethnic background population) and the fraction of that identified mutation in parent B's ethnic background population multiplied by (1 - mutation detection rate for parent A's ethnic background population) (i.e., $0.72 \times 0.28+0.54 \times 0.12$ ). The conditional probability that the neonate has one identifiable mutation if the neonate is a carrier due to a mutation derived from parent $\mathrm{A}$ or parent $\mathrm{B}$ is

Table 7

Bayesian analysis for a neonate born to non-Hispanic Caucasian and Hispanic Caucasian parents (Scenario 6$)^{a}$

\begin{tabular}{|c|c|c|c|c|c|c|c|}
\hline \multirow{3}{*}{$\begin{array}{l}\begin{array}{l}\text { Non-Hispanic Caucasian } \\
\text { parent A }\end{array} \\
\text { Prior probability } \\
\text { Hispanic parent B }\end{array}$} & & \multicolumn{3}{|c|}{ Carrier $(\mathrm{Da})$} & & \multicolumn{2}{|c|}{ Noncarrier } \\
\hline & & \multicolumn{3}{|c|}{$1 / 2$} & & \multicolumn{2}{|c|}{$1 / 2$} \\
\hline & \multicolumn{4}{|c|}{ Carrier (Db) } & Noncarrier & Carrier (Db) & Noncarrier \\
\hline Prior probability & \multicolumn{4}{|c|}{$1 / 46$} & $45 / 46$ & $1 / 46$ & $45 / 46$ \\
\hline \multicolumn{8}{|l|}{ Conditional probabilities } \\
\hline \multirow[t]{3}{*}{$\begin{array}{l}\text { Neonate with one } \\
\text { mutation detected }\end{array}$} & \multicolumn{2}{|c|}{ Affected $(\mathrm{Da}+\mathrm{Db})$} & Carrier & Carrier & \multirow[t]{2}{*}{$\begin{array}{l}\text { Carrier } \\
(\mathrm{Da})\end{array}$} & \multirow[t]{2}{*}{ Carrier $(\mathrm{Db})$} & \multirow[t]{2}{*}{$\cdots$} \\
\hline & & & $(\mathrm{Da})$ & $(\mathrm{Db})$ & & & \\
\hline & \multicolumn{2}{|c|}{$1 / 4$} & $1 / 4$ & $1 / 4$ & $1 / 2$ & $1 / 2$ & 0 \\
\hline Positive IRT test & \multicolumn{2}{|c|}{1} & 0.041 & 0.041 & 0.041 & 0.041 & 0.011 \\
\hline One mutation & $\mathrm{Da}$ & $\mathrm{Db}$ & $\mathrm{Da}$ & $\mathrm{Db}$ & $\mathrm{Da}$ & $\mathrm{Db}$ & $\cdots$ \\
\hline F508del identified & 0.72 & 0.54 & 0.72 & 0.54 & 0.72 & 0.54 & $\cdots$ \\
\hline \multirow{2}{*}{$\begin{array}{l}\text { No other mutation } \\
\text { detected }\end{array}$} & $\mathrm{Db}$ & $\mathrm{Da}$ & $\mathrm{N}$ & $\mathrm{N}$ & $\mathrm{N}$ & $\mathrm{N}$ & $\mathrm{N}$ \\
\hline & 0.28 & 0.12 & 1 & 1 & 1 & 1 & 1 \\
\hline Joint probability & $5.5 \times 10^{-4}$ & $1.8 \times 10^{-4}$ & $8.0 \times 10^{-5}$ & $6.0 \times 10^{-5}$ & $7.2 \times 10^{-3}$ & $1.2 \times 10^{-4}$ & 0 \\
\hline Posterior probability & 0.067 & 0.021 & 0.0098 & 0.0073 & 0.88 & 0.015 & 0 \\
\hline Column & A & B & $\mathrm{C}$ & $\mathrm{D}$ & $\mathrm{E}$ & F & $\mathrm{G}$ \\
\hline
\end{tabular}

${ }^{a}$ Neonate has been found to have one detectable mutation, F508del (heterozygous). The non-Hispanic Caucasian parent has an affected nephew who has not been tested. Neither parent has been tested. See explanation in the text.

$\mathrm{Da}$, disease allele derived from parent $\mathrm{A} ; \mathrm{Db}$, disease allele derived from parent $\mathrm{B} ; \mathrm{N}$, normal allele. 


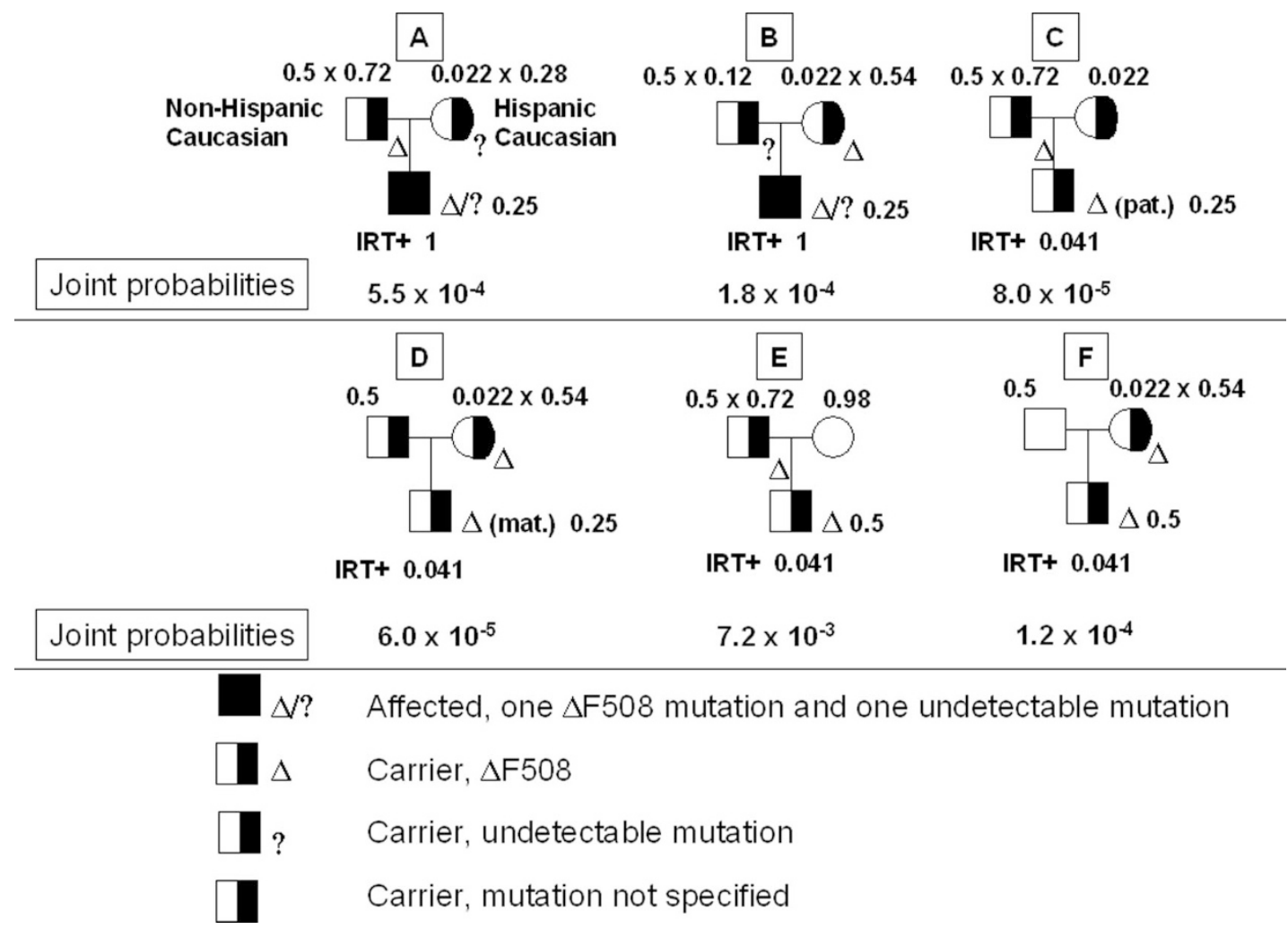

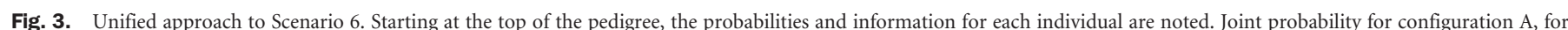

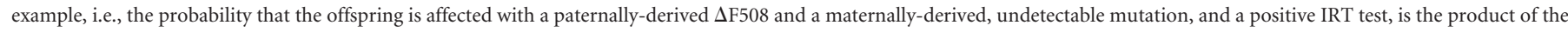

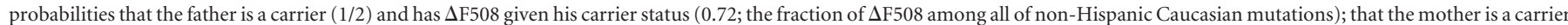

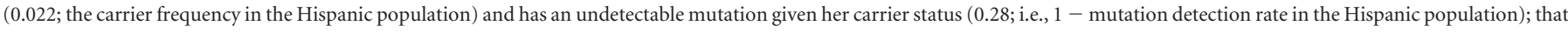
the neonate has inherited two mutations (1/4) given such carrier parents; and that the neonate tests positive for IRT given his or her affected status (1).

the fraction of that identified mutation in that parent's background population (i.e., 0.72 or 0.54 , respectively). The posterior probability that the neonate is affected is the sum of the posterior probabilities of columns A and B, or 0.088 .

\section{Scenario 7. Neonate of a Non-Hispanic Caucasian parent $A$ and a Hispanic American parent B, with a positive IRT test and no identifiable CFTR mutation; parent A with a family history of CF and negative CFTR carrier screening; parent B with no family history of CF and no carrier screening performed}

The Bayesian analysis for this scenario is shown in Table 8, which is based on Scenario 6 in Ogino et al. ${ }^{6}$ Suppose that parent $\mathrm{A}$ has an affected nephew who has not been tested. Before the IRT test and any CFTR mutation testing are performed, the probability that parent $A$ or parent $B$ is a CF carrier is $1 / 2$ or $1 / 46$, respectively. The conditional probability that parent $\mathrm{A}$ tests negative is $(1-$ mutation detection rate for parent A's ethnic background population). The conditional probability that the neonate has no identifiable mutation if affected is ( 1 - mutation detection rate for parent B's ethnic background population) $\times 1$, because parent $A$ tests negative and a mutation derived from parent $\mathrm{A}$, if present, is undetectable. The conditional probability that the neonate has no identifiable mutation if the neonate is a carrier with a mutation derived from parent A or parent $B$ is 1 or $(1$ - mutation de- tection rate for parent B's ethnic background population), respectively. The posterior probability that the neonate is affected is 0.013 . The posterior carrier probability for the neonate is the sum of the posterior probabilities of columns $B$, C, E, and G, or 0.18 .

\section{Scenario 8. Non-Hispanic Caucasian neonate with a positive IRT test and one identifiable CFTR mutation (F508del) present in parent A; parent B with no family history of CF and negative CFTR carrier screening}

Before the neonate was tested, the probability that parent $B$ is a carrier is 0.04 . The Bayesian analysis for this scenario is shown in Table 9, which is based on Scenario 4 in Ogino et al. ${ }^{6}$ The conditional probability that parent $\mathrm{B}$ tests negative is ( 1 mutation detection rate for parent B's ethnic background population). The conditional probability that the neonate has one identifiable mutation if affected is 1 , because a mutation derived from parent $\mathrm{A}$, if present, is identifiable, and a mutation derived from parent $B$, if present, is undetectable. The conditional probability that the neonate has one identifiable mutation, if the neonate is a carrier with a mutation derived from parent $\mathrm{A}$ or parent $\mathrm{B}$, is 1 or 0 , respectively, because $\mathrm{Db}$, if present, is undetectable. The posterior probability that the neonate is affected is 0.057 . 
Table 8

Bayesian analysis for a neonate born to non-Hispanic Caucasian and Hispanic Caucasian parents (Scenario 7) ${ }^{a}$

\begin{tabular}{|c|c|c|c|c|c|c|c|c|c|}
\hline $\begin{array}{l}\text { Non-Hispanic Caucasian } \\
\text { parent A }\end{array}$ & \multicolumn{6}{|c|}{ Carrier } & \multicolumn{3}{|c|}{ Noncarrier } \\
\hline Prior probability & \multicolumn{6}{|c|}{$1 / 2$} & \multicolumn{3}{|c|}{$1 / 2$} \\
\hline $\begin{array}{l}\text { Negative carrier } \\
\text { screening }\end{array}$ & \multicolumn{6}{|c|}{0.12} & \multicolumn{3}{|c|}{1} \\
\hline Hispanic parent B & \multicolumn{4}{|c|}{ Carrier } & \multicolumn{2}{|c|}{ Noncarrier } & \multicolumn{2}{|c|}{ Carrier } & Noncarrier \\
\hline Prior probability & \multicolumn{4}{|c|}{$1 / 46$} & \multicolumn{2}{|c|}{$45 / 46$} & \multicolumn{2}{|c|}{$1 / 46$} & $45 / 46$ \\
\hline \multicolumn{10}{|l|}{ Conditional probabilities } \\
\hline \multirow[t]{2}{*}{ Neonate (mutation) } & $\begin{array}{c}\text { Affected } \\
(\mathrm{Da}+\mathrm{Db})\end{array}$ & $\begin{array}{c}\text { Carrier } \\
(\mathrm{Da})\end{array}$ & $\begin{array}{c}\text { Carrier } \\
(\mathrm{Db})\end{array}$ & Noncarrier & $\begin{array}{c}\text { Carrier } \\
(\mathrm{Da})\end{array}$ & Noncarrier & $\begin{array}{c}\text { Carrier } \\
(\mathrm{Db})\end{array}$ & Noncarrier & Noncarrier \\
\hline & $1 / 4$ & $1 / 4$ & $1 / 4$ & $1 / 4$ & $1 / 2$ & $1 / 2$ & $1 / 2$ & $1 / 2$ & 1 \\
\hline Positive IRT test & 1 & 0.041 & 0.041 & 0.011 & 0.041 & 0.011 & 0.041 & 0.011 & 0.011 \\
\hline No mutation detected & 0.28 & 1 & 0.28 & 1 & 1 & 1 & 0.28 & 1 & 1 \\
\hline Joint probability & $9.1 \times 10^{-5}$ & $1.3 \times 10^{-5}$ & $3.7 \times 10^{-6}$ & $3.6 \times 10^{-6}$ & $1.2 \times 10^{-3}$ & $3.2 \times 10^{-4}$ & $6.2 \times 10^{-5}$ & $6.0 \times 10^{-5}$ & $5.4 \times 10^{-3}$ \\
\hline Posterior probability & 0.013 & 0.0019 & 0.00052 & 0.00050 & 0.17 & 0.045 & 0.0087 & 0.0084 & 0.75 \\
\hline Column & A & B & C & D & E & $\mathrm{F}$ & G & $\mathrm{H}$ & I \\
\hline
\end{tabular}

${ }^{a}$ No detectable mutation has been found either in the neonate, or in the non-Hispanic Caucasian parent A. The other Hispanic parent B has not been tested. Parent A has an affected nephew who has not been tested. See explanation in the text.

$\mathrm{Da}$, disease allele derived from parent $\mathrm{A} ; \mathrm{Db}$, disease allele derived from parent $\mathrm{B}$.

Table 9

Bayesian analysis for a non-Hispanic Caucasian neonate with one identifiable mutation present in Parent A (Scenario 8) ${ }^{a}$

\begin{tabular}{|c|c|c|c|c|}
\hline Parent B & & Carrier $(\mathrm{Db}+\mathrm{N})$ & & Noncarrier \\
\hline Prior probability & & 0.04 & & 0.96 \\
\hline \multicolumn{5}{|l|}{ Conditional probabilities } \\
\hline $\begin{array}{l}\text { Negative carrier screening } \\
\text { on parent } B\end{array}$ & & 0.12 & & 1 \\
\hline \multirow{2}{*}{$\begin{array}{l}\text { Neonate (affected or } \\
\text { carrier) }\end{array}$} & Affected $(\mathrm{Da}+\mathrm{Db})$ & Carrier $(\mathrm{Da}+\mathrm{N})$ & Carrier $(\mathrm{Db}+\mathrm{N})$ & Carrier $(\mathrm{Da}+\mathrm{N})$ \\
\hline & 0.25 & 0.25 & 0.25 & 0.5 \\
\hline Allele from parent A & $\mathrm{Da}$ (detected) & $\mathrm{Da}$ (detected) & $\mathrm{N}$ (no mutation) & $\mathrm{Da}$ (detected) \\
\hline \multirow[t]{2}{*}{ Allele from parent B } & $\mathrm{Db}$ (undetected) & $\mathrm{N}$ (no mutation) & $\mathrm{Db}$ (detected) & $\mathrm{N}$ (no mutation) \\
\hline & 1 & 1 & 0 & 1 \\
\hline Positive IRT test & 1 & 0.041 & 0.041 & 0.041 \\
\hline Joint probability & 0.0012 & 0.000049 & 0 & 0.020 \\
\hline Posterior probability & 0.057 & 0.0024 & 0 & 0.94 \\
\hline Column & A & $\mathrm{B}$ & $\mathrm{C}$ & $\mathrm{D}$ \\
\hline
\end{tabular}

${ }^{a}$ Assume that parent $\mathrm{A}$ is a carrier with a detectable mutation. Neonate has been found to have one detectable mutation (heterozygous) present in parent A. Parent B tests negative.

$\mathrm{Da}$, disease allele derived from parent $\mathrm{A} ; \mathrm{Db}$, disease allele derived from parent $\mathrm{B} ; \mathrm{N}$, normal allele.

\section{Scenario 9. Neonate with a positive IRT test, one or no identifiable} CFTR mutation (F508del), and a positive or negative sweat test

After neonatal screening with a positive IRT test and no detectable CFTR mutation, whether a sweat test is performed depends on the neonate's risk and other factors. If the IRT test is positive and one detectable mutation is present, the neonate typically undergoes confirmatory sweat testing. Sweat chloride levels in in- fants with CF, CF carrier infants, and noncarrier infants in Wisconsin were determined by Farrell and Koscik. ${ }^{18}$ The mean \pm 3 $\mathrm{SD}$ of sweat chloride in noncarrier infants $(N=184)$ and in carrier infants $(N=128)$ ranged from -5.0 to $26.2 \mathrm{mEq} / \mathrm{L}$ and from -10.3 to $40.1 \mathrm{mEq} / \mathrm{L}$, respectively. ${ }^{18}$ Mean \pm 3 SD of sweat chloride in $\mathrm{CF}$ infants with homozygous F508del $(N=61)$, in $\mathrm{CF}$ infants with one F508del and one non-F508del mutation $(N=$ 
47), and in CF infants with two non-F508del mutations $(N=7)$ ranged from 72.4 to $127.6 \mathrm{mEq} / \mathrm{L}$, from 52.0 to $143.2 \mathrm{mEq} / \mathrm{L}$, and from 57.3 to $131.9 \mathrm{mEq} / \mathrm{L}$, respectively. ${ }^{18}$ Therefore, when properly performed, sweat testing can discriminate $\mathrm{CF}$ infants from non-CF infants, with virtually no overlap of sweat chloride levels between these two groups. However, LeGrys's report ${ }^{19}$ of proficiency testing surveys on sweat analysis for CF demonstrated that some laboratories reported erroneous results. One should interpret sweat-test results cautiously, particularly when clinical findings are inconsistent with them, or when the sweat test is performed on neonates other than non-Hispanic Caucasians. To confirm that the sweat test can be a confirmatory test among ethnicities other than non-Hispanic Caucasians, ethnicity-specific sweat chloride levels among CF neonates, CF carrier neonates, and noncarrier neonates need to be determined. Sweat chloride levels does not seem to predict the severity of lung disease among CF patients. ${ }^{20}$

\section{DISCUSSION}

Bayesian analysis plays an essential role in calculations of genetic risk. ${ }^{2-5,21}$ We previously developed Bayesian methods to calculate autosomal recessive disease risks with only one or no detectable mutation, ${ }^{6}$ and methods to calculate CF disease and carrier risks in a variety of prenatal and carrier screening scenarios that provide common, difficult problems. ${ }^{7}$ These methods are particularly useful for CF. Using concrete examples, we have illustrated herein principles of genetic risk calculations for a variety of common scenarios that arise in CF neonatal screening.

Although prenatal screening and carrier screening for all couples have been advocated by the American College of Medical Genetics (ACMG) and the American College of Obstetrics and Gynecology (ACOG), 1,17,22 a large number of pregnancies and couples are not screened before birth, because of economic or other reasons. Neonatal screening is relatively easy to perform, and has been shown to be an effective way to prevent malnutrition and improve long-term growth of neonates affected with CF. 23

The assay characteristics of the IRT test that we used here were derived from a single large study on newborn screening by the IRT test, ${ }^{14}$ because this study is the only study available that allows calculations of the probability of a positive IRT test if a neonate is affected, a carrier, or a noncarrier. However, normal IRT value ranges may be different in different ethnic groups. Ethnicity-specific assay characteristics of the IRT test should be used when comprehensive data on IRT test results for CF affected, carrier, and noncarrier neonates in different ethnic groups are available in the future.

Effects of prenatal ultrasound screening, prenatal CFTR mutation testing, and neonatal screening on the birth prevalence of CF in Brittany, France have been investigated and an approximately $30 \%$ decrease in CF prevalence was estimated. ${ }^{24}$ Effects of public health measures on the frequencies of the various CFTR disease alleles need to be investigated, because they will impact risk assessments for CF considerably.
In conclusion, we present a variety of Bayesian methods to calculate CF risks for neonates with a positive IRT test. Our methods are applicable to many common clinical scenarios and allow CF disease risks to be calculated accurately, taking into account all relevant information. Our methods can also be applied to other autosomal recessive disorders and screening scenarios.

\section{NOTES ADDED IN PROOF}

1. In practice, when complicated scenarios are encountered, the calculations should always be checked independently by another geneticist to guard against errors; The authors abide by this rule. ${ }^{5}$

2. On March 8, 2005, The ACMG report entitled "Newborn Screening: Toward a Uniform Screening Panel and System" was submitted to the Health Resources and Services Administration of the U.S. Department of Health and Human Services, and made available for public comment. Among the 29 conditions for which there was a sufficient scientific and medical basis to recommend inclusion in newborn screening panels was cystic fibrosis. Its inclusion is similarly advocated by the March of Dimes and the Cystic Fibrosis Foundation.

\section{ACKNOWLEDGMENTS}

This project has been funded in whole or in part with Federal Funds from the National Cancer Institute, National Institutes of Health. We thank James Yankaskas and Michael Watson for helpful discussions.

\section{References}

1. Richards CS, Bradley LA, Amos J, Allitto B, Grody WW, Maddalena A et al. Standards and guidelines for CFTR mutation testing. Genet Med 2002;4:379-391.

2. Young ID. Introduction to risk calculation in genetic counseling, 2nd ed. Oxford: Oxford University Press, 1999.

3. Bridge PJ. The calculation of genetic risks: Worked examples in DNA diagnostics, 2nd ed. Baltimore: The Johns Hopkins University Press, 1997.

4. Ogino S, Wilson RB. Bayesian analysis and risk assessment in genetic counseling and testing. J Mol Diagn 2004;6:1-9.

5. Hodge SE, Flodman PL. Risk calculations: Still essential in the molecular age. Am J Med Genet 2004;129A:215-217.

6. Ogino S, Wilson RB, Grody WW. Bayesian risk assessment for autosomal recessive diseases: Fetal echogenic bowel with one or no detectable CFTR mutation. J Med Genet 2004;41:e70.

7. Ogino S, Wilson RB, Gold B, Hawley P, Grody WW. Bayesian analysis for cystic fibrosis risks in prenatal and carrier screening. Genet Med 2004;6:439-449.

8. Southern KW, Littlewood JM. Newborn screening programmes for cystic fibrosis. Paediatr Respir Rev 2003;4:299-305.

9. Bobadilla JL, Macek M, Jr., Fine JP, Farrell PM. Cystic fibrosis: A worldwide analysis of CFTR mutations-correlation with incidence data and application to screening. Hum Mutat 2002;19:575-606.

10. Watson MS, Cutting GR, Desnick RJ, Driscoll DA, Klinger K, Mennuti M et al. Cystic fibrosis population carrier screening: 2004 revision of american college of medical genetics mutation panel. Genet Med 2004;6:387-391.

11. Massie J, Du Sart D, Forshaw K, Carlin J, Forrest SM. The relationship between neonatal immunoreactive trypsinogen, deltaf508, and ivs8-5t. J Med Genet 2000;37:629-632.

12. Gregg RG, Simantel A, Farrell PM, Koscik R, Kosorok MR, Laxova A et al. Newborn screening for cystic fibrosis in Wisconsin: Comparison of biochemical and molecular methods. Pediatrics 1997;99:819-824.

13. Corbetta C, Seia M, Bassotti A, Ambrosioni A, Giunta A, Padoan R. Screening for cystic fibrosis in newborn infants: Results of a pilot programme based on a two tier protocol (irt/DNA/irt) in the Italian population. J Med Screen 2002;9:60-63.

14. Scotet V, De Braekeleer M, Audrezet MP, Lode L, Verlingue C, Quere I et al. Prevalence of CFTR mutations in hypertrypsinaemia detected through neonatal screening for cystic fibrosis. Clin Genet 2001;59:42-47. 
15. Hodge SE. A simple, unified approach to Bayesian risk calculations. J Genet Couns 1998;7:235-261.

16. Scotet V, de Braekeleer M, Roussey M, Rault G, Parent P, Dagorne M et al. Neonatal screening for cystic fibrosis in Brittany, France: Assessment of 10 years' experience and impact on prenatal diagnosis. Lancet 2000;356: $789-794$.

17. Grody WW, Cutting GR, Klinger KW, Richards CS, Watson MS, Desnick RJ. Laboratory standards and guidelines for population-based cystic fibrosis carrier screening. Genet Med 2001;3:149-154.

18. Farrell PM, Koscik RE. Sweat chloride concentrations in infants homozygous or heterozygous for f508 cystic fibrosis. Pediatrics 1996;97:524-528.

19. LeGrys VA. Sweat analysis proficiency testing for cystic fibrosis. Pediatr Pulmonol 2000;30:476-480.
20. Davis PB, Schluchter MD, Konstan MW. Relation of sweat chloride concentration to severity of lung disease in cystic fibrosis. Pediatr Pulmonol 2004;38:204-209.

21. Ogino $\mathrm{S}$, Wilson RB. Genetic testing and risk assessment for spinal muscular atrophy (SMA). Hum Genet 2002;111:477-500.

22. Richards CS, Grody WW. Prenatal screening for cystic fibrosis: Past, present and future. Expert Rev Mol Diagn 2004;4:49-62.

23. Farrell PM, Kosorok MR, Rock MJ, Laxova A, Zeng L, Lai HC et al. Early diagnosis of cystic fibrosis through neonatal screening prevents severe malnutrition and improves long-term growth: Wisconsin cystic fibrosis neonatal screening study group. Pediatrics 2001;107:1-13.

24. Scotet V, Audrezet MP, Roussey M, Rault G, Blayau M, De Braekeleer M et al Impact of public health strategies on the birth prevalence of cystic fibrosis in Brittany, France. Hum Genet 2003;113:280-285. 